\title{
Strong convergence theorems of Cesàro-type means for nonexpansive mapping in $\mathrm{CAT}(0)$ space
}

\author{
Jinfang Tang ${ }^{1}$, Shih-sen Chang ${ }^{2 *}$ and Jian Dong ${ }^{1}$
}

"Correspondence:
changss2013@163.com
2College of Statistics and
Mathematics, Yunnan University of
Finance and Economics, Kunming,
Yunnan 650221, China
Full list of author information is
available at the end of the article

available at the end of the article

\begin{abstract}
The iterative algorithms with Cesàro-type means for a nonexpansive mapping are proposed in CAT(0) spaces. Under suitable conditions, some strong convergence theorems for the sequence generated by the algorithms to a fixed point of the nonexpansive mapping are proved. We also proved that this fixed point is also a unique solution to some kind of variational inequality. The results presented in this paper extend and improve the corresponding results of some others.
\end{abstract}

Keywords: Cesàro-type means method; CAT(0) space; fixed point

\section{Introduction}

In 1975, Baillon [1] first proved the following nonlinear ergodic theorem.

Theorem 1.1 Suppose that $C$ is a nonempty closed convex subset of a Hilbert space $E$ and $T: C \rightarrow C$ is a nonexpansive mapping such that $F(T) \neq \emptyset$. Then, $\forall x \in C$, the Cesàro means

$$
T_{n} x=\frac{1}{n+1} \sum_{i=0}^{n} T^{i} x
$$

weakly converges to a fixed point of $T$.

In 1979, Bruck [2] generalized the Baillon Cesàro's means theorem from Hilbert space to uniformly convex Banach space with Fréchet differentiable norms.

In 2007, Song and Chen [3] defined the following viscosity iteration $\left\{x_{n}\right\}$ of the Cesàro means for nonexpansive mapping $T$ :

$$
x_{n+1}=\alpha_{n} f\left(x_{n}\right)+\left(1-\alpha_{n}\right) \frac{1}{n+1} \sum_{i=0}^{n} T^{i} x,
$$

and they proved that the sequence $\left\{x_{n}\right\}$ converged strongly to some point in $F(T)$ in a uniformly convex Banach space with weakly sequentially continuous duality mapping.

In 2009, Yao et al. [4] in a Banach space introduced the following process $\left\{x_{n}\right\}$ :

$$
x_{n+1}=\alpha_{n} u+\beta_{n} x_{n}+\gamma_{n} T x_{n}, \quad n \geq 0 .
$$

(C) 2015 Tang et al. This article is distributed under the terms of the Creative Commons Attribution 4.0 International License (http://creativecommons.org/licenses/by/4.0/), which permits unrestricted use, distribution, and reproduction in any medium, provided you give appropriate credit to the original author(s) and the source, provide a link to the Creative Commons license, and indicate if changes were made. 
They proved that the sequence $\left\{x_{n}\right\}$ converged strongly to a fixed point of $T$ under suitable control conditions of parameters.

In 2014, Zhu and Chen [5] proposed the following iterations with Cesàro's means for nonexpansive mappings:

$$
x_{n+1}=\alpha_{n} u+\beta_{n} x_{n}+\gamma_{n} \frac{1}{n+1} \sum_{i=0}^{n} T^{i} x_{n}, \quad n \geq 0,
$$

and viscosity iteration:

$$
x_{n+1}=\alpha_{n} f\left(x_{n}\right)+\beta_{n} x_{n}+\gamma_{n} \frac{1}{n+1} \sum_{i=0}^{n} T^{i} x_{n}, \quad n \geq 0 .
$$

They proved the sequences $\left\{x_{n}\right\}$ both converged strongly to a fixed point of $F(T)$ in the framework of a uniformly smooth Banach space.

As is well known, an extension of a linear version (usually in Banach spaces or Hilbert spaces) of this well-known result to a metric space has its own importance. The above iterative methods (1.1)-(1.5) involve general convex combinations. If we want to extend these results from Hilbert spaces or Banach spaces to metric spaces, we need some convex structure in a metric space to investigate their convergence on a nonlinear domain.

On the other hand, recently the theory and applications of CAT(0) space have been studied extensively by many authors.

Recall that a metric space $(X, d)$ is called a $\operatorname{CAT}(0)$ space, if it is geodesically connected and if every geodesic triangle in $X$ is at least as 'thin' as its comparison triangle in the Euclidean plane. It is known that any complete, simply connected Riemannian manifold having non-positive sectional curvature is a $\operatorname{CAT}(0)$ space. Other examples of CAT(0) spaces include pre-Hilbert spaces (see [6]), $R$-trees (see [7]), Euclidean buildings (see [8]), the complex Hilbert ball with a hyperbolic metric (see [9]), and many others. A complete $\mathrm{CAT}(0)$ space is often called a Hadamard space. A subset $K$ of a CAT(0) space $X$ is convex if, for any $x, y \in K$, we have $[x, y] \subset K$, where $[x, y]$ is the uniquely geodesic joining $x$ and $y$. For a thorough discussion of $\operatorname{CAT}(0)$ spaces and of the fundamental role they play in geometry, we refer the reader to Bridson and Haefliger [6].

Fixed point theory in CAT(0) spaces has been first studied by Kirk (see [10, 11]). He showed that every nonexpansive (single-valued) mapping defined on a bounded closed convex subset of a complete $\operatorname{CAT}(0)$ space always has a fixed point.

Motivated and inspired by the research going on in this direction, it is naturally to put forward the following.

Open question Can we extend the above Cesàro nonlinear ergodic theorems for nonexpansive mapping in [1-5] from a Hilbert space or Banach space to a CAT(0) space?

The purpose of this paper is to give an affirmative answer to this question. For solving this problem we first propose the following new iterations with Cesàro's means for nonexpansive mappings in the setting of $\mathrm{CAT}(0)$ spaces:

$$
x_{n+1}=\alpha_{n} u \oplus \beta_{n} x_{n} \oplus \gamma_{n}\left(\bigoplus_{i=0}^{n} \frac{1}{n+1} T^{i} x_{n}\right),
$$


and the viscosity iteration:

$$
x_{n+1}=\alpha_{n} f\left(x_{n}\right) \oplus \beta_{n} x_{n} \oplus \gamma_{n}\left(\bigoplus_{i=0}^{n} \frac{1}{n+1} T^{i} x_{n}\right) \text {, }
$$

and then study the strong convergence of the iterative sequences (1.6) and (1.7). Under suitable conditions, some strong converge theorems to a fixed point of the nonexpansive mapping are proved. We also prove that this fixed point is a unique solution of some kind of variational inequality in $\mathrm{CAT}(0)$ spaces. The results presented in this paper are new; they extend and improve corresponding previous results.

Next we give some examples of iteration (1.6) or (1.7) with Cesàro's means for nonexpansive mappings to illustrate the generation of our new iterations.

Example 1 If $X$ is a real Hilbert (or Banach space), and if $\alpha_{n}=0, \beta_{n}=0, \forall n \geq 1$, then the iteration (1.6) with Cesàro's means for nonexpansive mappings is reduced as the same iteration of Baillon [1] (or Bruck [2]).

Example 2 If $X$ is a real Banach space and $n=1$, then the iteration (1.6) with Cesàro's means for nonexpansive mappings is reduced to the same iteration of Yao et al. [4]. If $n \geq 2$, then the iteration (1.6) can be written

$$
x_{n+1}=\alpha_{n} u+\beta_{n} x_{n}+\gamma_{n}\left(\sum_{i=0}^{n} \frac{1}{n+1} T^{i} x_{n}\right),
$$

which is a generalization of results due to Yao et al. [4].

Example 3 The iterations (1.6) and (1.7) are a generalization of the iterations of Zhu and Chen [5] from Banach space to CAT(0) spaces.

Example 4 Let $E=\mathbb{R}$ with the usual metric, $T$ be a nonexpansive mapping defined by $T x=\sin x$, and $f$ be a contractive mapping defined by $f(x)=\frac{x}{2}$. Let $\alpha_{n}=\frac{1}{2 n}, \beta_{n}=\frac{(4 n-3)}{4 n}$, and $\gamma_{n}=\frac{1}{4 n}, \forall n \geq 1$. Then the iteration (1.6) and (1.7) with Cesàro's means for nonexpansive mappings $T x=\sin x$ are reduced to the following iterations, respectively:

$$
\begin{aligned}
& x_{n+1}=\frac{1}{2 n} u+\frac{4 n-3}{4 n} x_{n}+\frac{1}{4 n(n+1)}\left(x_{n}+T x_{n}+T^{2} x_{n}+\cdots+T^{n} x_{n}\right), \\
& x_{n+1}=\frac{1}{2 n} \cdot \frac{1}{2} x_{n}+\frac{4 n-3}{4 n} x_{n}+\frac{1}{4 n(n+1)}\left(x_{n}+T x_{n}+T^{2} x_{n}+\cdots+T^{n} x_{n}\right) .
\end{aligned}
$$

Example 5 If $(X, d)$ is a $\operatorname{CAT}(0)$ space, $n=1$ and $\beta_{n}=0$, then the iteration (1.7) with Cesàro's means for nonexpansive mappings is reduced to the following iteration, with Cesàro's means for nonexpansive mappings:

$$
x_{n+1}=\alpha_{n} f\left(x_{n}\right) \oplus \frac{\gamma_{n}}{2} x_{n} \oplus \frac{\gamma_{n}}{2} T x_{n}
$$

which is a generalization of the iterations in Wangkeeree and Preechasilp [12] from a Banach space to $\mathrm{CAT}(0)$ spaces. 


\section{Preliminaries and lemmas}

In this paper, we write $(1-t) x \oplus t y$ for the unique point $z$ in the geodesic segment joining from $x$ to $y$ such that

$$
d(x, z)=t d(x, y), \quad d(y, z)=(1-t) d(x, y) .
$$

Lemma 2.1 [13] A geodesic space $(X, d)$ is a $C A T(0)$ space, if and only if the inequality

$$
d^{2}((1-t) x \oplus t y, z) \leq(1-t) d^{2}(x, z)+t d^{2}(y, z)-t(1-t) d^{2}(x, y)
$$

is satisfied for all $x, y, z \in X$ and $t \in[0,1]$. In particular, if $x, y, z$ are points in a $C A T(0)$ space and $t \in[0,1]$, then

$$
d((1-t) x \oplus t y, z) \leq(1-t) d(x, z)+t d(y, z) .
$$

Lemma 2.2 [6] Let $(X, d)$ be a $C A T(0)$ space, $p, q, r, s \in X$, and $\lambda \in[0,1]$. Then

$$
d(\lambda p \oplus(1-\lambda) q, \lambda r \oplus(1-\lambda) s) \leq \lambda d(p, r)+(1-\lambda) d(q, s) .
$$

By induction, we write

$$
\bigoplus_{m=1}^{n} \lambda_{m} x_{m}:=\left(1-\lambda_{n}\right)\left(\frac{\lambda_{1}}{1-\lambda_{n}} x_{1} \oplus \frac{\lambda_{2}}{1-\lambda_{n}} x_{2} \oplus \cdots \oplus \frac{\lambda_{n-1}}{1-\lambda_{n}} x_{n-1}\right) \oplus \lambda_{n} x_{n} .
$$

Lemma 2.3 [14] Let $(X, d)$ be a $C A T(0)$ space, then for any sequence $\left\{\lambda_{i}\right\}_{i=1}^{n}$ in $[0,1]$ satisfying $\sum_{i=1}^{n} \lambda_{i}=1$ and for any $\left\{x_{i}\right\}_{i=1}^{n} \subset X$, the following conclusions hold:

$$
d\left(\bigoplus_{i=1}^{n} \lambda_{i} x_{i}, x\right) \leq \sum_{i=1}^{n} \lambda_{i} d\left(x_{i}, x\right), \quad x \in X
$$

and

$$
d^{2}\left(\bigoplus_{i=1}^{n} \lambda_{i} x_{i}, x\right) \leq \sum_{i=1}^{n} \lambda_{i} d^{2}\left(x_{i}, x\right)-\lambda_{1} \lambda_{2} d^{2}\left(x_{1}, x_{2}\right), \quad x \in X .
$$

Lemma 2.4 Let $\left\{x_{i}\right\}$ and $\left\{y_{i}\right\}$ be any sequences of a CAT(0) space $X$, then for any sequence $\left\{\lambda_{i}\right\}_{i=1}^{k}$ in $[0,1]$ satisfying $\sum_{i=1}^{k} \lambda_{i}=1$ and for any $\left\{x_{i}\right\}_{i=1}^{k} \subset X$, the following inequality holds:

$$
d\left(\bigoplus_{i=1}^{k} \lambda_{i} x_{i}, \bigoplus_{i=1}^{k} \lambda_{i} y_{i}\right) \leq \sum_{i=1}^{k} \lambda_{i} d\left(x_{i}, y_{i}\right)
$$

Proof It is obvious that (2.6) holds for $k=2$. Suppose that (2.6) holds for some $k \geq 3$. Next we prove that (2.6) is also true for $k+1$. From (2.3) and (2.4) we have 


$$
\begin{aligned}
& d\left(\bigoplus_{i=1}^{k+1} \lambda_{i} x_{i}, \bigoplus_{i=1}^{k+1} \lambda_{i} y_{i}\right) \\
& \quad=d\left(\left(1-\lambda_{k+1}\right)\left(\bigoplus_{i=1}^{k} \frac{\lambda_{i}}{1-\lambda_{k+1}} x_{i}\right) \oplus \lambda_{k+1} x_{k+1},\left(1-\lambda_{k+1}\right)\left(\bigoplus_{i=1}^{k} \frac{\lambda_{i}}{1-\lambda_{k+1}} y_{i}\right) \oplus \lambda_{k+1} y_{k+1}\right) \\
& \quad \leq\left(1-\lambda_{k+1}\right) d\left(\bigoplus_{i=1}^{k} \frac{\lambda_{i}}{1-\lambda_{k+1}} x_{i}, \bigoplus_{i=1}^{k} \frac{\lambda_{i}}{1-\lambda_{k+1}} y_{i}\right)+\lambda_{k+1} d\left(x_{k+1}, y_{k+1}\right) \\
& \leq\left(1-\lambda_{k+1}\right) \sum_{i=1}^{k} \frac{\lambda_{i}}{1-\lambda_{k+1}} d\left(x_{i}, y_{i}\right)+\lambda_{k+1} d\left(x_{k+1}, y_{k+1}\right) \\
& \quad=\sum_{i=1}^{k+1} \lambda_{i} d\left(x_{i}, y_{i}\right) .
\end{aligned}
$$

This implies that (2.6) holds.

Berg and Nikolaev [15] introduced the concept of quasilinearization as follows. Let us denote a pair $(a, b) \in X \times X$ by $\overrightarrow{a b}$ and call it a vector. Then quasilinearization is defined as a map $\langle\cdot, \cdot\rangle:(X \times X) \times(X \times X) \rightarrow \mathbb{R}$ defined by

$$
\langle\overrightarrow{a b}, \overrightarrow{c d}\rangle=\frac{1}{2}\left(d^{2}(a, d)+d^{2}(b, c)-d^{2}(a, c)-d^{2}(b, d)\right) \quad(a, b, c, d \in X)
$$

It is easy to seen that $\langle\overrightarrow{a b}, \overrightarrow{c d}\rangle=\langle\overrightarrow{c d}, \overrightarrow{a b}\rangle,\langle\overrightarrow{a b}, \overrightarrow{c d}\rangle=-\langle\overrightarrow{b a}, \overrightarrow{c d}\rangle$, and $\langle\overrightarrow{a x}, \overrightarrow{c d}\rangle+\langle\overrightarrow{x b}, \overrightarrow{c d}\rangle=$ $\langle\overrightarrow{a b}, \overrightarrow{c d}\rangle$ for all $a, b, c, d \in X$. We say that $X$ satisfies the Cauchy-Schwarz inequality if

$$
\langle\overrightarrow{a b}, \overrightarrow{c d}\rangle \leq d(a, b) d(c, d)
$$

for all $a, b, c, d \in X$. It is well known [15] that a geodesically connected metric space is a CAT(0) space if and only if it satisfies the Cauchy-Schwarz inequality.

Let $C$ be a nonempty closed convex subset of a complete $C A T(0)$ space $X$. The metric projection $P_{C}: X \rightarrow C$ is defined by

$$
u=P_{C}(x) \quad \Leftrightarrow \quad d(u, x)=\inf \{d(y, x): y \in C\}, \quad \forall x \in X .
$$

Lemma 2.5 [16] Let $C$ be a nonempty convex subset of a complete $C A T(0)$ space $X, x \in X$, and $u \in C$. Then $u=P_{C}(x)$ if and only if

$$
\langle\overrightarrow{y u}, \overrightarrow{u x}\rangle \geq 0, \quad \forall y \in C
$$

Lemma 2.6 ([12]) Let $C$ be a closed convex subset of a complete $C A T(0)$ space $X$, and let $T: C \rightarrow C$ be a nonexpansive mapping with $F(T) \neq \emptyset$. Let $f$ be a contraction on $C$ with coefficient $\alpha<1$. For each $t \in[0,1]$, let $\left\{x_{t}\right\}$ be the net defined by

$$
x_{t}=t f\left(x_{t}\right) \oplus(1-t) T x_{t} .
$$


Then $\lim _{t \rightarrow 0} x_{t}=\tilde{x}$, some point $F(T)$ which is the unique solution of the following variational inequality:

$$
\langle\overrightarrow{\tilde{x} f(\tilde{x})}, \vec{x} \tilde{x}\rangle \geq 0, \quad \forall x \in F(T)
$$

Lemma 2.7 [12] Let $X$ be a complete $C A T(0)$ space. Then, for any $u, x, y \in X$, the following inequality holds:

$$
d^{2}(x, u) \leq d^{2}(y, u)+2\langle\overrightarrow{x y}, \overrightarrow{x u}\rangle .
$$

Lemma 2.8 [12] Let $X$ be a complete $C A T(0)$ space. For any $t \in[0,1]$ and $u, v \in X$, let $u_{t}=t u \oplus(1-t) v$. Then, for any $x, y \in X$,

(i) $\left\langle\overrightarrow{u_{t} x}, \overrightarrow{u_{t} y}\right\rangle \leq t\left\langle\overrightarrow{u_{x}}, \overrightarrow{u_{t} y}\right\rangle+(1-t)\left\langle\overrightarrow{v x}, \overrightarrow{u_{t} y}\right\rangle$;

(ii) $\left\langle\overrightarrow{u_{t} x}, \overrightarrow{u y}\right\rangle \leq t\langle\overrightarrow{u x}, \overrightarrow{u y}\rangle+(1-t)\langle\overrightarrow{v x}, \overrightarrow{u y}\rangle$, and $\left\langle\overrightarrow{u_{t} x}, \overrightarrow{v y}\right\rangle \leq t\langle\overrightarrow{u x}, \overrightarrow{v y}\rangle+(1-t)\langle\overrightarrow{v x}, \overrightarrow{v y}\rangle$.

Lemma 2.9 [17] Let $\left\{a_{n}\right\}$ be a sequence of non-negative real numbers satisfying the property $a_{n+1} \leq\left(1-\alpha_{n}\right) a_{n}+\alpha_{n} \beta_{n}, n \geq 0$, where $\left\{\alpha_{n}\right\} \subset(0,1)$ and $\left\{\beta_{n}\right\} \subset \mathbb{R}$ such that

(i) $\sum_{n=0}^{\infty} \alpha_{n}=\infty$;

(ii) $\lim \sup _{n \rightarrow \infty} \beta_{n} \leq 0$ or $\sum_{n=0}^{\infty}\left|\alpha_{n} \beta_{n}\right|<\infty$.

Then $\left\{a_{n}\right\}$ converges to zero as $n \rightarrow \infty$.

\section{Approximative iterative algorithms}

Throughout this section, we assume that $C$ is a nonempty and closed convex subset of a complete CAT(0) space $X$, and $T: C \rightarrow C$ is a nonexpansive mappings with $F(T) \neq \emptyset$. Let $f$ be a contraction on $C$ with coefficient $k \in(0,1)$. Suppose $\left\{\alpha_{n}\right\},\left\{\beta_{n}\right\}$, and $\left\{\gamma_{n}\right\}$ are three real sequences in $(0,1)$ satisfying

(i) $\alpha_{n}+\beta_{n}+\gamma_{n}=1$, for all $n \geq 0$;

(ii) $\lim _{n \rightarrow \infty} \alpha_{n}=0$ and $\sum_{n=0}^{\infty} \alpha_{n}=\infty$;

(iii) $\lim _{n \rightarrow \infty} \gamma_{n}=0$.

In the following, we first present two important results.

The first one is to prove the mapping $S:=\bigoplus_{i=0}^{n} \frac{1}{n+1} T^{i}: C \rightarrow C$ is nonexpansive: In fact, for any $x, y \in C$, from Lemma 2.4 we get

$$
\begin{aligned}
d(S x, S y) & =d\left(\bigoplus_{i=0}^{n} \frac{1}{n+1} T^{i} x, \bigoplus_{i=0}^{n} \frac{1}{n+1} T^{i} y\right) \\
& \leq \sum_{i=0}^{n} \frac{1}{n+1} d\left(T^{i} x, T^{i} y\right) \\
& \leq d(x, y),
\end{aligned}
$$

i.e., $S$ is nonexpansive.

The second one is to prove the following mapping $T_{t, n}: C \rightarrow C$ is contractive:

$$
T_{t, n} x=\frac{\left(1-\alpha_{n}\right) t}{\gamma_{n}+t \beta_{n}} f(x) \oplus \frac{(1-t) \gamma_{n}}{\gamma_{n}+t \beta_{n}}\left(\bigoplus_{i=0}^{n} \frac{1}{n+1} T^{i} x\right) .
$$


In fact, the mapping $T_{t, n}$ can be written as

$$
T_{t, n} x=\lambda_{n} f(x) \oplus\left(1-\lambda_{n}\right)\left(\bigoplus_{i=0}^{n} \frac{1}{n+1} T^{i} x\right), \quad x \in C,
$$

where $\lambda_{n}=\frac{\left(1-\alpha_{n}\right) t}{\gamma_{n}+t \beta_{n}}$. Therefore this kind of mappings has just a similar form to (2.9). Hence for any $x, y \in C$, from Lemma 2.4 , we have

$$
\begin{aligned}
d & \left(T_{t, n}(x), T_{t, n}(y)\right) \\
& =d\left(\lambda_{n} f(x) \oplus\left(1-\lambda_{n}\right) \bigoplus_{i=0}^{n} \frac{1}{n+1} T^{i} x, \lambda_{n} f(y) \oplus\left(1-\lambda_{n}\right) \bigoplus_{i=0}^{n} \frac{1}{n+1} T^{i} y\right) \\
& \leq \lambda_{n} d(f(x), f(y))+\left(1-\lambda_{n}\right) d\left(\bigoplus_{i=0}^{n} \frac{1}{n+1} T^{i} x, \bigoplus_{i=0}^{n} \frac{1}{n+1} T^{i} y\right) \\
& \leq \lambda_{n} k d(x, y)+\frac{1-\lambda_{n}}{n+1} \sum_{i=0}^{n} d\left(T^{i} x, T^{i} y\right) \\
& \leq \lambda_{n} k d(x, y)+\left(1-\lambda_{n}\right) d(x, y) \\
& =\left(1-\lambda_{n}(1-k)\right) d(x, y),
\end{aligned}
$$

i.e., $T_{t, n}$ is a contractive mapping. Hence, it has a unique fixed point (denote by $z_{t, n}$ ), i.e.,

$$
z_{t, n}=\lambda_{n} f\left(z_{t, n}\right) \oplus\left(1-\lambda_{n}\right)\left(\bigoplus_{i=0}^{n} \frac{1}{n+1} T^{i}\left(z_{t, n}\right)\right) .
$$

From Lemma 2.6, for each given $n \geq 1$, we have

$$
\lim _{t \rightarrow 0} z_{t, n}=x_{n} \in F\left(\bigoplus_{i=0}^{n} \frac{1}{n+1} T^{i}\right)
$$

and it is the unique solution of the following variational inequality:

$$
\left\langle\overrightarrow{x_{n} f\left(x_{n}\right)}, \overrightarrow{x x_{n}}\right\rangle \geq 0, \quad \forall x \in F\left(\bigoplus_{i=0}^{n} \frac{1}{n+1} T^{i}\right) .
$$

Next we prove that for each $n \geq 1$

$$
F(T)=F\left(\bigoplus_{i=0}^{n} \frac{1}{n+1} T^{i}\right) .
$$

If fact, if $x \in F(T)$, it is obvious that $x \in F\left(\bigoplus_{i=0}^{n} \frac{1}{n+1} T^{i}\right)$, for each $n \geq 1$. This implies that $F(T) \subset F\left(\bigoplus_{i=0}^{n} \frac{1}{n+1} T^{i}\right)$ for each $n \geq 1$.

By using induction, now we prove that

$$
F\left(\bigoplus_{i=0}^{n} \frac{1}{n+1} T^{i}\right) \subset F(T) \quad \text { for each } n \geq 1 .
$$


Indeed, if $n=1$, and $x \in F\left(\bigoplus_{i=0}^{1} \frac{1}{2} T^{i}\right)$, i.e., $x=\frac{1}{2} x \oplus \frac{1}{2} T x$, then we have

$$
\frac{1}{2} x \oplus \frac{1}{2} x=\frac{1}{2} x \oplus \frac{1}{2} T x
$$

Since the geodesic segment joining any two points in CAT(0) space is unique, it follows from (3.5) that $x=T x$, i.e., $x \in F(T)$.

If (3.4) is true for some $n \geq 2$, now we prove that (3.4) is also true for $n+1$.

Indeed, if $x \in F\left(\bigoplus_{i=0}^{n+1} \frac{1}{n+2} T^{i}\right)$, i.e.,

$$
x=\bigoplus_{i=0}^{n+1} \frac{1}{n+2} T^{i} x
$$

It is easy to see that (3.6) can be rewritten as

$$
\frac{n+1}{n+2} x \oplus \frac{1}{n+2} x=\frac{n+1}{n+2}\left\{\frac{x}{n+1} \oplus \frac{T x}{n+1} \oplus \cdots \oplus \frac{T^{n} x}{n+1}\right\} \oplus \frac{1}{n+2} T^{n+1} x
$$

By the same reasoning showing that the geodesic segment joining any two points in $\mathrm{CAT}(0)$ space is unique, from (3.7) we have

$$
\frac{n+1}{n+2} x=\frac{n+1}{n+2}\left\{\frac{x}{n+1} \oplus \frac{T x}{n+1} \oplus \cdots \oplus \frac{T^{n} x}{n+1}\right\} \quad \text { and } \quad \frac{1}{n+2} x=\frac{1}{n+2} T^{n+1} x
$$

This implies that

$$
x=\frac{x}{n+1} \oplus \frac{T x}{n+1} \oplus \cdots \oplus \frac{T^{n} x}{n+1} \quad \text { and } \quad x=T^{n+1} x .
$$

By the assumption of induction, from (3.8) we have $x=T x$. The conclusion (3.4) is proved. Therefore the conclusion (3.3) is also proved.

By using (3.3), (3.1) can be written as

$$
\lim _{t \rightarrow 0} z_{t, n}=x_{n} \in F(T)
$$

and $x_{n}$ is the unique solution of the following variational inequality:

$$
\left\langle\overrightarrow{x_{n} f\left(x_{n}\right)}, \overrightarrow{x x_{n}}\right\rangle \geq 0, \quad \forall x \in F(T)
$$

Next we prove that for any positive integers $m, n, x_{n}=x_{m}$ (where $x_{n}$ is the limit in (3.9)). Therefore (3.9) can be written as

$$
\lim _{t \rightarrow 0} z_{t, n}=x_{n}=\tilde{x} \in F(T)
$$

where $\tilde{x}$ is some point, and it is the unique solution of the following variational inequality:

$$
\langle\overrightarrow{\tilde{x} f(\tilde{x})}, \overrightarrow{x \tilde{x}}\rangle \geq 0, \quad \forall x \in F(T) .
$$


In fact, it follows from (3.10) that

$$
\begin{aligned}
& \left\langle\overrightarrow{x_{n} f\left(x_{n}\right)}, \overrightarrow{x x_{n}}\right\rangle \geq 0, \quad \forall x \in F(T), \\
& \left\langle\overrightarrow{x_{m} f\left(x_{m}\right)}, \overrightarrow{y x_{m}}\right\rangle \geq 0, \quad \forall y \in F(T) .
\end{aligned}
$$

Taking $x=x_{m}$ in the first inequality and $y=x_{n}$ in the second inequality and then adding up the resultant two inequalities, we have

$$
\begin{aligned}
0 \leq & \left|\overrightarrow{x_{n} f\left(x_{n}\right)}, \overrightarrow{x_{m} x_{n}}\right\rangle-\left\langle\overrightarrow{x_{m} f\left(x_{m}\right)}, \overrightarrow{x_{m} x_{n}}\right\rangle \\
\leq & \left.\left\langle\overrightarrow{x_{n} f\left(x_{m}\right.}\right), \overrightarrow{x_{m} x_{n}}\right\rangle+\left\langle\overrightarrow{f\left(x_{m}\right) f\left(x_{n}\right)}, \overrightarrow{x_{m} x_{n}}\right\rangle \\
& -\left\langle\overrightarrow{x_{m} x_{n}}, \overrightarrow{x_{m} x_{n}}\right\rangle-\left\langle\overrightarrow{x_{n} f\left(x_{m}\right)}, \overrightarrow{x_{m} x_{n}}\right\rangle \\
= & \left|\overrightarrow{f\left(x_{m}\right) f\left(x_{n}\right)}, \overrightarrow{x_{m} x_{n}}\right\rangle-\left\langle\overrightarrow{x_{m} x_{n}}, \overrightarrow{x_{m} x_{n}}\right\rangle \\
\leq & d\left(f\left(x_{m}\right), f\left(x_{n}\right)\right) d\left(x_{m}, x_{n}\right)-d^{2}\left(x_{m}, x_{n}\right) \\
\leq & k d^{2}\left(x_{m}, x_{n}\right)-d^{2}\left(x_{m}, x_{n}\right)=(k-1) d^{2}\left(x_{m}, x_{n}\right) .
\end{aligned}
$$

Since $k \in(0,1)$, this implies that $d\left(x_{m}, x_{n}\right)=0$, i.e., $x_{m}=x_{n}, \forall m, n \geq 1$.

The conclusion is proved.

We are now in a position to propose the iterative algorithms with Cesàro's means for a nonexpansive mapping in a complete $\mathrm{CAT}(0)$ space $X$, and prove that the sequence generated by the algorithms converges strongly to a fixed point of the nonexpansive mapping which is also a unique solution of some kind of variational inequality.

Theorem 3.1 Let $C$ be a closed convex subset of a complete $C A T(0)$ space $X$, and $T: C \rightarrow C$ be a nonexpansive mapping with $F(T) \neq \emptyset$. Let $f$ be a contraction on $C$ with coefficient $k \in\left(0, \frac{1}{2}\right)$. Suppose $x_{0} \in C$ and the sequence $\left\{x_{n}\right\}$ is given by

$$
x_{n+1}=\alpha_{n} f\left(x_{n}\right) \oplus \beta_{n} x_{n} \oplus \gamma_{n}\left(\bigoplus_{i=0}^{n} \frac{1}{n+1} T^{i} x_{n}\right) \text {, }
$$

where $T^{0}=I,\left\{\alpha_{n}\right\},\left\{\beta_{n}\right\}$, and $\left\{\gamma_{n}\right\}$ are three real sequences in $(0,1)$ satisfying conditions (i)(iii). Then $\left\{x_{n}\right\}$ converges strongly to $\tilde{x}$ such that $\tilde{x}=P_{F(T)} f(\tilde{x})$, which is equivalent to the following variational inequality:

$$
\langle\overrightarrow{\tilde{x} f(\tilde{x})}, \overrightarrow{x \tilde{x}}\rangle \geq 0, \quad \forall x \in F(T) .
$$

Proof We first show that the sequence $\left\{x_{n}\right\}$ is bounded. For any $p \in F(T)$, we have

$$
\begin{aligned}
d\left(x_{n+1}, p\right) & =d\left(\alpha_{n} f\left(x_{n}\right) \oplus \beta_{n} x_{n} \oplus \gamma_{n}\left(\bigoplus_{i=0}^{n} \frac{1}{n+1} T^{i} x_{n}\right), p\right) \\
& \leq \alpha_{n} d\left(f\left(x_{n}\right), p\right)+\beta_{n} d\left(x_{n}, p\right)+\gamma_{n} d\left(\bigoplus_{i=0}^{n} \frac{1}{n+1} T^{i} x_{n}, p\right) \\
& \leq \alpha_{n}\left(d\left(f\left(x_{n}\right), f(p)\right)+d(f(p), p)\right)+\beta_{n} d\left(x_{n}, p\right)+\gamma_{n} \sum_{i=0}^{n} \frac{1}{n+1} d\left(T^{i} x_{n}, T^{i} p\right)
\end{aligned}
$$




$$
\begin{aligned}
& \leq \alpha_{n} k d\left(x_{n}, p\right)+\alpha_{n} d(f(p), p)+\beta_{n} d\left(x_{n}, p\right)+\gamma_{n} d\left(x_{n}, p\right) \\
& =\left(1-\alpha_{n}(1-k)\right) d\left(x_{n}, p\right)+\alpha_{n}(1-k) \cdot \frac{1}{1-k} d(f(p), p) \\
& \leq \max \left\{d\left(x_{n}, p\right), \frac{1}{1-k} d(f(p), p)\right\} .
\end{aligned}
$$

By induction, we have

$$
d\left(x_{n}, p\right) \leq \max \left\{d\left(x_{0}, p\right), \frac{1}{1-k} d(f(p), p)\right\}
$$

for all $n \geq 0$. Hence $\left\{x_{n}\right\}$ is bounded, and so are $\left\{f\left(x_{n}\right)\right\}$ and $\left\{T^{i}\left(x_{n}\right)\right\}$.

Let $\left\{z_{t, n}\right\}$ be a sequence in $C$ such that

$$
z_{t, n}=\frac{\left(1-\alpha_{n}\right) t}{\gamma_{n}+t \beta_{n}} f\left(z_{t, n}\right) \oplus \frac{(1-t) \gamma_{n}}{\gamma_{n}+t \beta_{n}}\left(\bigoplus_{i=0}^{n} \frac{1}{n+1} T^{i} z_{t, n}\right) .
$$

It follows from (3.11) that $\left\{z_{t, n}\right\}$ converges strongly to a fixed point $\tilde{x} \in F(T)$, which is also a unique solution of the variational inequality (3.14).

Now we claim that

$$
\limsup _{n \rightarrow \infty}\left|\overrightarrow{f(\tilde{x}) \tilde{x}}, \overrightarrow{x_{n+1}} \overrightarrow{\tilde{x}}\right\rangle \leq 0
$$

It follows from Lemma 2.8 and Lemma 2.4 that

$$
\begin{aligned}
& d^{2}\left(z_{t, n}, x_{n+1}\right) \\
& =\left\langle\overrightarrow{z_{t, n} x_{n+1}}, \overrightarrow{z_{t, n} x_{n+1}}\right\rangle
\end{aligned}
$$

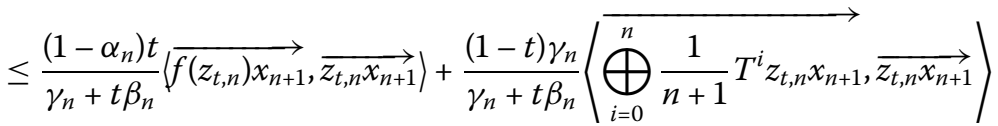

$$
\begin{aligned}
& =\frac{\left(1-\alpha_{n}\right) t}{\gamma_{n}+t \beta_{n}}\left[\left\langle\overrightarrow{f\left(z_{t, n}\right) f(\tilde{x})}, \overrightarrow{z_{t, n} x_{n+1}}\right\rangle+\left\langle\overrightarrow{f(\tilde{x}) \tilde{x}}, \overrightarrow{z_{t, n} x_{n+1}}\right\rangle\right. \\
& \left.+\left\langle\overrightarrow{\tilde{x} z_{t, n}}, \overrightarrow{z_{t, n} x_{n+1}}\right\rangle+\left\langle\overrightarrow{z_{t, n} x_{n+1}}, \overrightarrow{z_{t, n} x_{n+1}}\right\rangle\right] \\
& +\frac{(1-t) \gamma_{n}}{\gamma_{n}+t \beta_{n}}\left[\left\langle\bigoplus_{i=0}^{n} \frac{1}{n+1} T^{i} z_{t, n} \bigoplus_{i=0}^{n} \frac{1}{n+1} T^{i} x_{n+1}, \overrightarrow{z_{t, n} x_{n+1}}\right\rangle\right. \\
& \left.+\left\langle\bigoplus_{i=0}^{n} \frac{1}{n+1} T^{i} x_{n+1} x_{n+1}, \overline{z_{t, n} x_{n+1}}\right\rangle\right] \\
& \leq \frac{\left(1-\alpha_{n}\right) t}{\gamma_{n}+t \beta_{n}}\left[k d\left(z_{t, n}, \tilde{x}\right) d\left(z_{t, n}, x_{n+1}\right)+\left\langle\overrightarrow{f(\tilde{x}) \tilde{x},}, \overrightarrow{z_{t, n} x_{n+1}}\right\rangle\right. \\
& \left.+d\left(\tilde{x}, z_{t, n}\right) d\left(z_{t, n}, x_{n+1}\right)+d^{2}\left(z_{t, n}, x_{n+1}\right)\right] \\
& +\frac{(1-t) \gamma_{n}}{\gamma_{n}+t \beta_{n}}\left[d\left(\bigoplus_{i=0}^{n} \frac{1}{n+1} T^{i} z_{t, n}, \bigoplus_{i=0}^{n} \frac{1}{n+1} T^{i} x_{n+1}\right) d\left(z_{t, n}, x_{n+1}\right)\right.
\end{aligned}
$$




$$
\begin{aligned}
& \left.+d\left(\bigoplus_{i=0}^{n} \frac{1}{n+1} T^{i} x_{n+1}, x_{n+1}\right) d\left(z_{t, n}, x_{n+1}\right)\right] \\
\leq & \frac{\left(1-\alpha_{n}\right) t}{\gamma_{n}+t \beta_{n}}\left[k d\left(z_{t, n}, \tilde{x}\right) d\left(z_{t, n}, x_{n+1}\right)+\left\langle\overrightarrow{f(\tilde{x}) \tilde{x},} \overrightarrow{z_{t, n} x_{n+1}}\right\rangle\right. \\
& \left.+d\left(\tilde{x}, z_{t, n}\right) d\left(z_{t, n}, x_{n+1}\right)+d^{2}\left(z_{t, n}, x_{n+1}\right)\right] \\
& +\frac{(1-t) \gamma_{n}}{\gamma_{n}+t \beta_{n}}\left[d^{2}\left(z_{t, n}, x_{n+1}\right)+\frac{1}{n+1} \sum_{i=0}^{n} d\left(T^{i} x_{n+1}, x_{n+1}\right) d\left(z_{t, n}, x_{n+1}\right)\right] \\
\leq & \frac{\left(1-\alpha_{n}\right) t}{\gamma_{n}+t \beta_{n}}\left[M k d\left(z_{t, n}, \tilde{x}\right)+\left\langle\overrightarrow{f(\tilde{x}) \tilde{x},} \overrightarrow{z_{t, n} x_{n+1}}\right\rangle+M d\left(\tilde{x}, z_{t, n}\right)\right] \\
& +d^{2}\left(z_{t, n}, x_{n+1}\right)+\frac{(1-t) \gamma_{n} M N}{\gamma_{n}+t \beta_{n}},
\end{aligned}
$$

where

$$
M \geq \sup \left\{d\left(z_{t, n}, x_{n+1}\right), n \geq 0,0<t<1\right\}, \quad N \geq \sup \left\{d\left(T^{i} x_{n+1}, x_{n+1}\right), n \geq 0, i \geq 0\right\} .
$$

This implies that

$$
\left\langle\overrightarrow{f(\tilde{x}) \tilde{x}}, \overrightarrow{x_{n+1} z_{t, n}}\right\rangle \leq(1+k) M d\left(z_{t, n}, \tilde{x}\right)+\frac{(1-t) \gamma_{n} M N}{\left(1-\alpha_{n}\right) t} .
$$

Taking the upper limit as $n \rightarrow \infty$ first, and then taking the upper limit as $t \rightarrow 0$, we get

$$
\limsup _{t \rightarrow 0} \limsup _{n \rightarrow \infty}\left(\overrightarrow{f(\tilde{x})} \vec{x}, \overrightarrow{x_{n+1} z_{t, n}}\right) \leq 0
$$

Since

$$
\begin{aligned}
\left\langle\overrightarrow{f(\tilde{x}) \tilde{x}}, \overrightarrow{x_{n+1}} \vec{x}\right\rangle & =\left\langle\overrightarrow{f(\tilde{x}) \tilde{x}}, \overrightarrow{x_{n+1} z_{t, n}}\right\rangle+\left\langle\overrightarrow{f(\tilde{x}) \tilde{x}}, \overrightarrow{z_{t, n} \tilde{x}}\right\rangle \\
& \leq\left\langle\overrightarrow{f(\tilde{x}) \tilde{x}}, \overrightarrow{x_{n+1} z_{t, n}}\right\rangle+d(f(\tilde{x}), \tilde{x}) d\left(z_{t, n}, \tilde{x}\right) .
\end{aligned}
$$

Thus, by taking the upper limit as $n \rightarrow \infty$ first, and then taking the upper limit as $t \rightarrow 0$, it follows from $z_{t, n} \rightarrow \tilde{x}$ and (3.17) that

$$
\limsup _{t \rightarrow 0} \limsup _{n \rightarrow \infty}\left|\overrightarrow{f(\tilde{x}) \tilde{x}}, \overrightarrow{x_{n+1}} \vec{x}\right\rangle \leq 0
$$

Hence

$$
\limsup _{n \rightarrow \infty}\left|\overrightarrow{f(\tilde{x})} \vec{x}, \overrightarrow{x_{n+1}} \vec{x}\right\rangle \leq 0 .
$$

Finally, we prove that $x_{n} \rightarrow \tilde{x}$ as $n \rightarrow \infty$. In fact, for any $n \geq 0$, let

$$
\left\{\begin{array}{l}
u_{n}:=\alpha_{n} \tilde{x} \oplus\left(1-\alpha_{n}\right) y_{n} \\
y_{n}:=\frac{\beta_{n}}{1-\alpha_{n}} x_{n} \oplus \frac{\gamma_{n}}{1-\alpha_{n}}\left(\bigoplus_{i=0}^{n} \frac{1}{n+1} T^{i} x_{n}\right) .
\end{array}\right.
$$


From Lemma 2.7 and Lemma 2.8 we have

$$
\begin{aligned}
& d^{2}\left(x_{n+1}, \tilde{x}\right) \\
& \leq d^{2}\left(u_{n}, \tilde{x}\right)+2\left\langle\overrightarrow{x_{n+1} u_{n}}, \overrightarrow{x_{n+1}} \overrightarrow{\tilde{x}}\right\rangle \\
& \leq\left(1-\alpha_{n}\right)^{2} d^{2}\left(y_{n}, \tilde{x}\right)+2\left[\alpha_{n}\left\langle\overrightarrow{f\left(x_{n}\right) u_{n}}, \overrightarrow{x_{n+1} \tilde{x}}\right\rangle+\left(1-\alpha_{n}\right)\left\langle\overrightarrow{y_{n} u_{n}}, \overrightarrow{x_{n+1}} \overrightarrow{\tilde{x}}\right\rangle\right] \\
& \leq\left(1-\alpha_{n}\right)^{2} d^{2}\left(x_{n}, \tilde{x}\right)+2\left[\alpha_{n}^{2} \mid \overrightarrow{f\left(x_{n}\right)} \vec{x}, \overrightarrow{x_{n+1}} \vec{x}\right) \\
& \left.+\alpha_{n}\left(1-\alpha_{n}\right)\left\langle\overrightarrow{f\left(x_{n}\right) y_{n}}, \overrightarrow{x_{n+1}} \overrightarrow{\tilde{x}}\right\rangle+\alpha_{n}\left(1-\alpha_{n}\right)\left\langle\overrightarrow{y_{n} \tilde{x}}, \overrightarrow{x_{n+1} \tilde{x}}\right\rangle+\left(1-\alpha_{n}\right)^{2}\left\langle\overrightarrow{y_{n} y_{n}}, \overrightarrow{x_{n+1}} \tilde{\tilde{x}}\right\rangle\right] \\
& =\left(1-\alpha_{n}\right)^{2} d^{2}\left(x_{n}, \tilde{x}\right)+2\left[\alpha_{n}^{2}\left|\overrightarrow{f\left(x_{n}\right)} \vec{x}, \overrightarrow{x_{n+1}} \vec{x}\right\rangle+\alpha_{n}\left(1-\alpha_{n}\right)\left|\overrightarrow{f\left(x_{n}\right) \tilde{x}}, \overrightarrow{x_{n+1}} \overrightarrow{\tilde{x}}\right\rangle\right] \\
& =\left(1-\alpha_{n}\right)^{2} d^{2}\left(x_{n}, \tilde{x}\right)+2 \alpha_{n}\left\langle\overrightarrow{f\left(x_{n}\right) \tilde{x}}, \overrightarrow{x_{n+1}} \vec{x}\right\rangle \\
& =\left(1-\alpha_{n}\right)^{2} d^{2}\left(x_{n}, \tilde{x}\right)+2 \alpha_{n}\left|\overrightarrow{f\left(x_{n}\right) f(\tilde{x})}, \overrightarrow{x_{n+1}} \vec{x}\right\rangle+2 \alpha_{n}\left|\overrightarrow{f(\tilde{x})} \vec{x}, \overrightarrow{x_{n+1}} \overrightarrow{\tilde{x}}\right\rangle \\
& \leq\left(1-\alpha_{n}\right)^{2} d^{2}\left(x_{n}, \tilde{x}\right)+2 \alpha_{n} k d\left(x_{n}, \tilde{x}\right) d\left(x_{n+1}, \tilde{x}\right)+2 \alpha_{n}\left\langle\overrightarrow{f(\tilde{x})} \vec{x}, \overrightarrow{x_{n+1}} \vec{x}\right\rangle \\
& \leq\left(1-\alpha_{n}\right)^{2} d^{2}\left(x_{n}, \tilde{x}\right)+\alpha_{n} k\left(d^{2}\left(x_{n}, \tilde{x}\right)+d^{2}\left(x_{n+1}, \tilde{x}\right)\right)+2 \alpha_{n}\left\langle\overrightarrow{f(\tilde{x}) \tilde{x}}, \overrightarrow{x_{n+1}} \overrightarrow{\tilde{x}}\right\rangle .
\end{aligned}
$$

This implies that

$$
\begin{aligned}
d^{2}\left(x_{n+1}, \tilde{x}\right) & \leq \frac{1-(2-k) \alpha_{n}+\alpha_{n}^{2}}{1-\alpha_{n} k} d^{2}\left(x_{n}, \tilde{x}\right)+\frac{2 \alpha_{n}}{1-\alpha_{n} k}\left\langle\overrightarrow{f(\tilde{x}) \tilde{x}}, \overrightarrow{x_{n+1}} \overrightarrow{\tilde{x}}\right\rangle \\
& =\left(1-\frac{\alpha_{n}\left(2-2 k-\alpha_{n}\right)}{1-\alpha_{n} k}\right) d^{2}\left(x_{n}, \tilde{x}\right)+\frac{2 \alpha_{n}}{1-\alpha_{n} k}\left\langle\overrightarrow{f(\tilde{x}) \tilde{x}}, \overrightarrow{x_{n+1} \tilde{x}}\right\rangle
\end{aligned}
$$

Then it follows that

$$
d^{2}\left(x_{n+1}, \tilde{x}\right) \leq\left(1-\alpha_{n}^{\prime}\right) d^{2}\left(x_{n}, \tilde{x}\right)+\alpha_{n}^{\prime} \beta_{n}^{\prime},
$$

where

$$
\alpha_{n}^{\prime}=\frac{\alpha_{n}\left(2-2 k-\alpha_{n}\right)}{1-\alpha_{n} k}, \quad \beta_{n}^{\prime}=\frac{2}{2-2 k-\alpha_{n}}\left\langle\overrightarrow{f(\tilde{x}) \tilde{x}}, \overrightarrow{x_{n+1} \tilde{x}}\right\rangle
$$

Applying Lemma 2.9, we can conclude that $x_{n} \rightarrow \tilde{x}$ as $n \rightarrow \infty$. This completes the proof.

Letting $f\left(x_{n}\right) \equiv u$ for all $n \in \mathbb{N}$, the following theorem can be obtained from Theorem 3.1 immediately.

Theorem 3.2 Let $C$ be a closed convex subset of a complete CAT(0) space $X$, and let $T$ : $C \rightarrow C$ be a nonexpansive mapping satisfying $F(T) \neq \emptyset$. For given $x_{0} \in C$ arbitrarily and fixed point $u \in C$, the sequence $\left\{x_{n}\right\}$ is given by

$$
x_{n+1}=\alpha_{n} u \oplus \beta_{n} x_{n} \oplus \gamma_{n}\left(\bigoplus_{i=0}^{n} \frac{1}{n+1} T^{i} x_{n}\right)
$$


where $T^{0}=I,\left\{\alpha_{n}\right\},\left\{\beta_{n}\right\}$, and $\left\{\gamma_{n}\right\}$ are three real sequences in $(0,1)$ satisfying conditions (i)-(iii). Then $\left\{x_{n}\right\}$ converges strongly to $\tilde{x}$ such that $\tilde{x}=P_{F(T)} u$, which is equivalent to the following variational inequality:

$$
\langle\overrightarrow{\tilde{x} u}, \overrightarrow{x \tilde{x}}\rangle \geq 0, \quad \forall x \in F(T)
$$

Remark 3.3 Theorem 3.1 and Theorem 3.2 are two new results. The proofs are simple and different from many others which extend the Cesàro nonlinear ergodic theorems for nonexpansive mappings in Baillon [1], Bruck [2], Song and Chen [3], Zhu and Chen [5], Yao et al. [4] from Hilbert space or Banach space to CAT(0) spaces. Theorem 3.1 is also a generalization of the results by Wangkeeree and Preechasilp [12].

\section{Competing interests}

The authors declare that they have no competing interests.

\section{Authors' contributions}

All the authors contributed equally to the writing of the present article. They also read and approved the final paper.

\section{Author details}

${ }^{1}$ Department of Mathematics, Yibin University, Yibin, Sichuan 644007, China. ${ }^{2}$ College of Statistics and Mathematics, Yunnan University of Finance and Economics, Kunming, Yunnan 650221, China.

\section{Acknowledgements}

The authors would like to express their thanks to the editors and the referees for their helpful suggestions and advises. This work was supported by the Scientific Research Fund of Sichuan Provincial Department of Science and Technology (2015JY0165) and the Scientific Research Fund of Sichuan Provincial Education Department (14ZA0271) and the Scientific Research Project of Yibin University (2013YY06). This work was also supported by the National Natural Science Foundation of China (Grant No. 11361070).

Received: 18 March 2015 Accepted: 15 June 2015 Published online: 01 July 2015

\section{References}

1. Baillon, JB: Un théorème de type ergodique pour les contractions non linéairs dans un espaces de Hilbert. C. R. Acad. Sci. Paris Sér. A-B 280(22), 1511-1514 (1975)

2. Bruck, RE: A simple proof of the mean ergodic theorem for nonlinear contractions in Banach spaces. Isr. J. Math. 32(2-3), 107-116 (1979)

3. Song, Y, Chen, R: Viscosity approximate methods to Cesàro means for non-expansive mappings. Appl. Math. Comput. 186(2), 1120-1128 (2007)

4. Yao, Y, Liou, YC, Zhou, H: Strong convergence of an iterative method for nonexpansive mappings with new control conditions. Nonlinear Anal., Theory Methods Appl. 70(6), 2332-2336 (2009)

5. Zhu, Z, Chen, R: Strong convergence on iterative methods of Cesàro means for nonexpansive mapping in Banach space. Abstr. Appl. Anal. 2014, Article ID 205875 (2014)

6. Bridson, MR, Haefliger, A: Metric Spaces of Non-positive Curvature. Grundlehren der Mathematischen Wissenschaften, vol. 319. Springer, Berlin (1999)

7. Kirk, WA: Fixed point theory in CAT(0) spaces and R-trees. Fixed Point Theory Appl. 2004(4), 309-316 (2004)

8. Brown, KS (ed.): Buildings. Springer, New York (1989)

9. Goebel, K, Reich, S: Uniform Convexity, Hyperbolic Geometry, and Nonexpansive Mappings. Monograph and Textbooks in Pure and Applied Mathematics, vol. 83. Dekker, New York (1984)

10. Kirk, WA: Geodesic geometry and fixed point theory. In: Seminar of Mathematical Analysis (Malaga/Seville, 2002/2003). Colección Abierta, vol. 64, pp. 195-225. University of Seville Secretary of Publications, Seville (2003)

11. Kirk, WA: Geodesic geometry and fixed point theory. II. In: International Conference on Fixed Point Theory and Applications, pp. 113-142. Yokohama Publishers, Yokohama (2004)

12. Wangkeeree, R, Preechasilp, P: Viscosity approximation methods for nonexpansive semigroups in CAT(0) spaces. Fixed Point Theory Appl. 2013, Article ID 160 (2013). doi:10.1186/1687-1812-2013-160

13. Dhompongsa, S, Panyanak, B: On $\Delta$-convergence theorems in CAT(0) spaces. Comput. Math. Appl. 56(10), 2572-2579 (2008)

14. Tang, JF: Viscosity approximation methods for a family of nonexpansive mappings in CAT(0) spaces. Abstr. Appl. Anal. 2014, Article ID 389804 (2014)

15. Berg, ID, Nikolaev, IG: Quasilinearization and curvature of Alexandrov spaces. Geom. Dedic. 133, 195-218 (2008)

16. Dehghan, H, Rooin, J: A characterization of metric projection in CAT(0) spaces. In: International Conference on Functional Equation, Geometric Functions and Applications (ICFGA 2012), 10-12th May 2012, pp. 41-43. Payame Noor University, Tabriz (2012)

17. Xu, HK: An iterative approach to quadratic optimization. J. Optim. Theory Appl. 116, 659-678 (2003) 